\title{
Communications
}

Chemical Biology

DOI: 10.1002/anie.200904510

\section{BTB-1: A Small Molecule Inhibitor of the Mitotic Motor Protein Kif18A***}

Mario Catarinella, Tamara Grüner, Tobias Strittmatter, Andreas Marx, and Thomas U. Mayer*

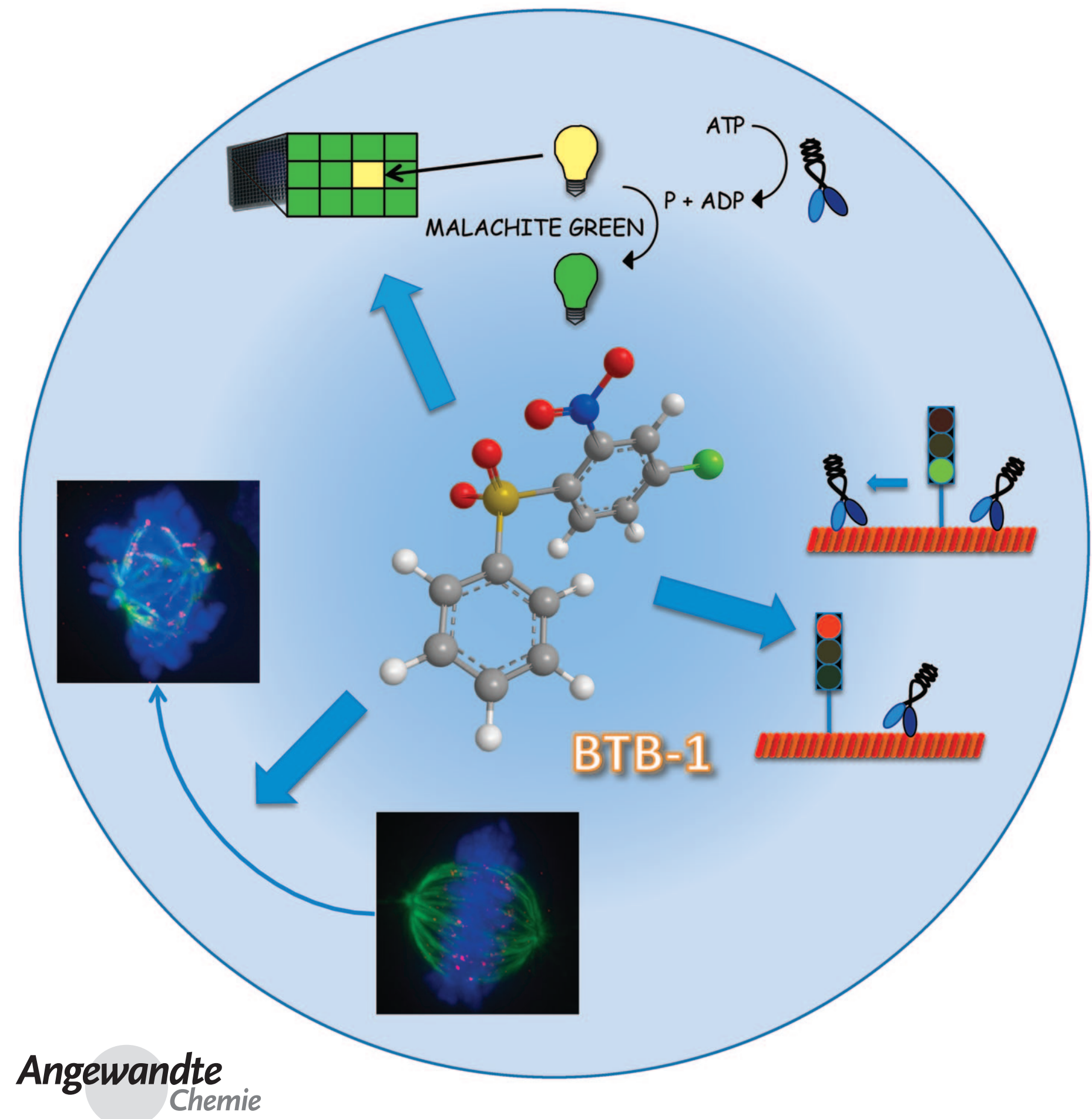


The survival and development of each organism relies on the equal partitioning of its genome during cell division. Errors in this process can lead to severe developmental defects and cancer in humans. Key for the accurate distribution of the genome is the mitotic spindle composed of dynamic microtubules (Mts). ${ }^{[1]}$ The shape and function of the mitotic spindle depends on the concerted action of various kinesins, these are molecular motor proteins which as microtubule-stimulated ATPases convert chemical energy into mechanical force. Recently, we identified the kinesin- 8 member Kif18A as a central component for the correct alignment of chromosomes at the spindle equator. ${ }^{[2]}$ Furthermore, in vitro analyses revealed that Kif18A distinguishes itself from all other kinesins by its dual functionality: motility and depolymerase activity. ${ }^{[2-4]}$ Owing to their fast and often reversible mode of action, small molecules are ideally suited to dissect the function and mechanism of proteins. Given the complex enzymatic characteristics of Kif18A, we set up a smallmolecule screen to identify inhibitors of Kif18A. Herein we report the discovery of BTB-1 (Figure 1a), the first smallmolecule inhibitor of Kif18A. We show that BTB-1 potently inhibits the ATPase activity of Kif18A $\left(\mathrm{IC}_{50}=1.69 \mu \mathrm{M}\right)$ but not of other tested key mitotic kinesins. BTB-1 blocks the motility of Kif18A in a reversible manner. Notably, BTB-1 inhibits Kif18A in an adenosine triphosphate(ATP)-competitive but microtubule-uncompetitive manner and slows down the progression of cells through mitosis.

To identify Kif18A inhibitors, we screened a 9000 member library of diverse small molecules for compounds that inhibit in vitro the ATPase activity of the recombinant motordomain of Kif18A (GST-Kif18A ${ }^{\text {motor }}$; GST = glutathion-S-transferase). The release of free phosphate as determined by a malachite green-based assay was used as readout for the

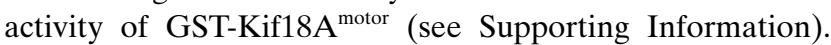
Compounds were screened in duplicate at a concentration of about $50 \mu \mathrm{M}$ and considered as "hits" if they inhibited the Kif $18 \mathrm{~A}^{\text {motor }}$-mediated ATP hydrolysis by more than $65 \%$. Of the identified four hits, BTB-1 (Figure 1a) was the most potent and selective and, thus, selected for further analyses. To determine the $\mathrm{IC}_{50}$ we used an enzyme-coupled assay to measure the rate of ATP hydrolysis by recombinant HisKif18 $\mathrm{A}^{\text {motor }}$ in the presence of Mts and increasing concentrations of BTB-1 or DMSO as solvent control (see Supporting Information). BTB-1 greatly inhibited the Mt-stimulated

[*] M. Catarinella, T. Strittmatter, Prof. Dr. A. Marx, Prof. Dr. T. U. Mayer Konstanz Research School Chemical Biology

University of Konstanz

78457 Konstanz (Germany)

Fax: (+ 49) 7531-88-3707

E-mail: Thomas.u.Mayer@uni-konstanz.de

Homepage: http://www.uni-konstanz.de/thomasmayer/

T. Grüner

London Research Institute, Lincoln's Inn Fields Laboratories WC2A3PX London (UK)

$[* *$ We gratefully acknowledge funding by the DAAD and Marie Curie Actions 6. We also thank Prof. Dr. W. Hofer and the members of the Mayer lab for helpful discussions.

64 Supporting information for this article is available on the WWW under http://dx.doi.org/10.1002/anie.200904510.

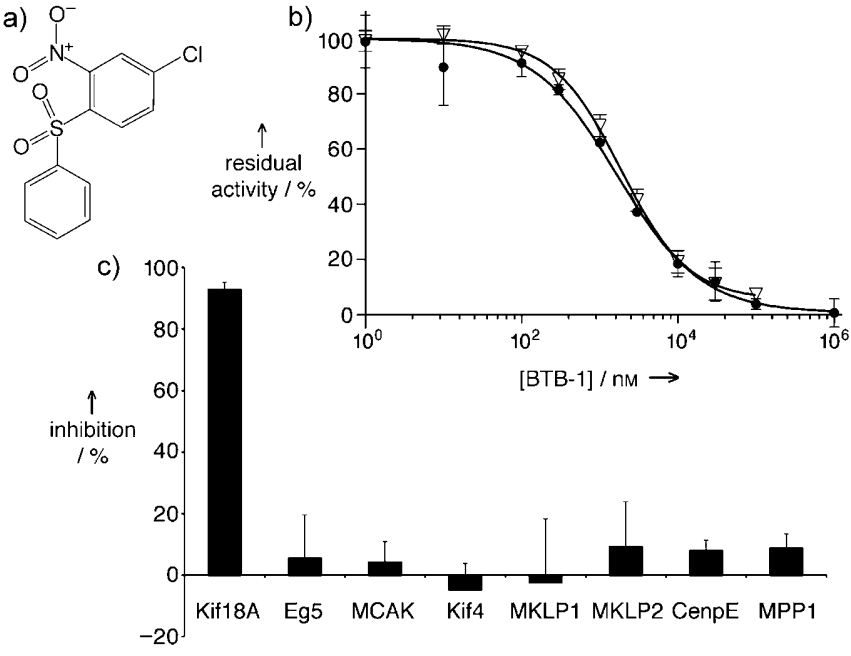

Figure 1. a) Structure of the identified compound BTB-1. b) Increasing concentrations of BTB-1 $(\bullet)$ and re-synthesized BTB-1 $(\nabla)$ were used to estimate the inhibitor $I_{50}$. c) Inhibitory effect of $100 \mu \mathrm{M} B$ BT-1 tested on different recombinant kinesins by enzyme-coupled assay (see Supporting Information). Averages of three independent experiments and standard deviations are shown.

ATPase activity of His-Kif18A ${ }^{\text {motor }}$ with an $\mathrm{IC}_{50}$ of $1.69 \mu \mathrm{M}$ (Figure 1b). Thus, BTB-1 is the first inhibitor for Kif18A, a key enzyme essential for proper chromosome segregation in eukaryotes ranging from yeast to human. To confirm the chemical identity of BTB-1, the molecule was re-synthesized (see Supporting Information). The re-synthesized BTB-1 was equally potent in inhibiting His-Kif18A ${ }^{\text {motor }}\left(\mathrm{IC}_{50}=1.86 \mu \mathrm{m}\right.$; Figure $1 \mathrm{~b})$. Next, we addressed the specificity of BTB-1. Importantly, our studies showed that $100 \mu \mathrm{M}$ BTB-1 did not significantly inhibit any of the other tested mitotic kinesins (Figure 1c).

To gain insights into the mode of action of BTB-1, we analyzed whether BTB-1 affects Kif18A in a reversible manner. To this end, we established an in vitro Mt gliding assay using a flow chamber which allows us to quickly exchange the solution of the reaction. The movement of Mts was recorded by time-lapse microscopy and displayed by a kymograph in which the position of an individual $\mathrm{Mt}$ (horizontal axis) is plotted as a function of time (vertical axis) as shown in Figure 2. In the presence of DMSO, recombinant full-length Kif18A (His-Kif18A ${ }^{\mathrm{FL}}$ ) immobilized on the surface of the glass slide moved fluorescently labeled microtubules at a speed of $(0.036 \pm 0.015) \mu \mathrm{m} \mathrm{min}^{-1}$, consistent with previous reports ${ }^{[2]}$ (Figure $2 \mathrm{~b}, \mathrm{c}$, and Movie S1 in the Supporting Information). Flushing-in $100 \mu \mathrm{M}$ BTB-1 almost completely stopped the His-Kif18A ${ }^{\mathrm{FL}}$-dependent Mt motility (Figure 2b,c and Movie S2 in the Supporting Information). This effect was specific because $100 \mu \mathrm{m}$ monastrol, a selective inhibitor of the mitotic kinesin Eg5, ${ }^{[5]}$ did not affect HisKif18A $\mathrm{A}^{\mathrm{FL}}$-dependent motility (Figure $2 \mathrm{~d}$, e and Movie S4 in the Supporting Information). Upon wash-out of BTB-1, HisKif18 $\mathrm{A}^{\mathrm{FL}}$ regained most of its activity and moved Mts at $(0.027 \pm 0.013) \mu \mathrm{mmin}^{-1}$ (Figure 2b,c and Movie S3 in the Supporting Information). Thus, BTB-1 is a reversible inhibitor of Kif18A. 
a)

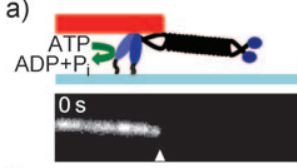

b)

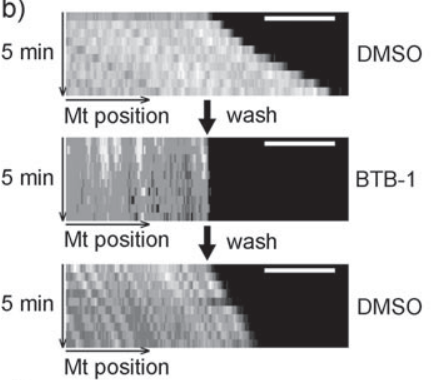

d)

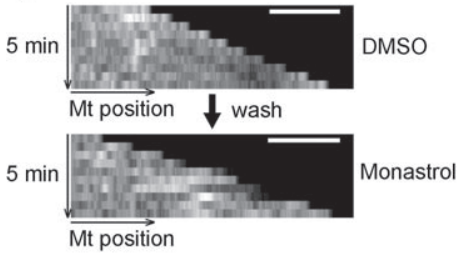

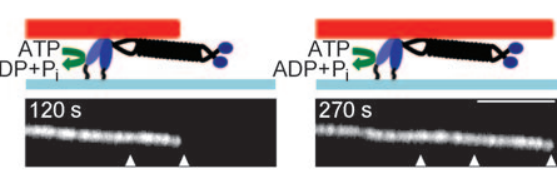

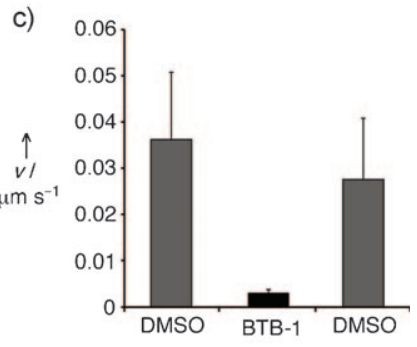

e)

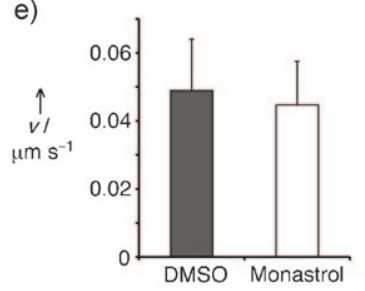

Figure 2. a) Recombinant His-Kif18 $\mathrm{A}^{\mathrm{FL}}$ was adsorbed to the glass surface and incubated with rhodamine-labeled Mts. Upon ATP hydrolysis, Kif18A is able to induce Mts gliding. Top: schematic representation of the assay ( $\mathrm{red}=\mathrm{Mt}$, blue/black structure $=\mathrm{Kif1} 8 \mathrm{~A}$, light blue $=$ glass surface, $\mathrm{P}_{\mathrm{i}}=$ phosphate). Bottom: Fluorescence images of Kif18Amediated microtubule movement (arrows indicate the microtubule tip at each time point; scale bar $=5 \mu \mathrm{m}$ ). b) Representative kymographs of $\mathrm{Mt}$ gliding assay performed in the presence of DMSO, after flushing in $100 \mu \mathrm{M}$ BTB-1, and after wash-out of BTB-1 (see text for details and Supporting Information, Movies S1-S3). c) Quantification of Mt motility $(n=10 \mathrm{Mts}$, averages of three independent experiments and standard deviations are shown). d) Representative kymographs of HisKif1 $8 \mathrm{~A}^{\mathrm{FL}}$-mediated movement of a $\mathrm{Mt}$ in the presence of DMSO or $100 \mu \mathrm{M}$ monastrol (see Supporting Information, Movie S4). e) Quantification of Mt motility ( $n=10 \mathrm{Mts}$, averages of three independent experiments and standard deviations are shown).

Kinesins hydrolyze ATP as they walk along microtubules, this shows ATP to be a genuine substrate and microtubules as pseudosubstrates that are not turned over by the enzyme. We investigated whether BTB-1 competes with ATP for Kif18A binding. Specifically, we determined the rate of Mt-stimulated ATP hydrolysis by His-Kif18A ${ }^{\text {motor }}$ in the presence of saturating concentrations of Mts, varying concentrations of ATP and BTB-1, and fitted each set of data to the MichaelisMenten equation (see Supporting Information). As can be derived from Figure $3 \mathrm{a}$, BTB-1 increased the $K_{\mathrm{m}}$ value for ATP while not significantly affecting the $V_{\max }$ value. ATP $\gamma \mathrm{S}$, a non-hydrolyzable ATP-competitive analogue, similarly affected $K_{\mathrm{m}}$ and $V_{\max }$ (Figure $3 \mathrm{~b}$ ) implying that BTB-1 inhibits Kif18A in an ATP-competitive manner.

Next, we analyzed how microtubules affect the inhibitory effect of BTB-1 on Kif18A. To this end, the rate of HisKif18 ${ }^{\text {motor }}$-mediated ATP hydrolysis in the presence of saturating ATP concentrations and varying concentrations of Mts and BTB-1 was quantified (Figure 3c). BTB-1 affected both the $K_{1 / 2}$ value for Mts (for Mts as pseudosubstrates the term $K_{1 / 2}$ instead of $K_{\mathrm{m}}$ is used) and the $V_{\max }$ value indicating

that BTB-1 might act in a noncompetitive, uncompetitive, or mixed-competition manner. Detailed analyses of the Michaelis-Menten kinetics identified the uncompetitive mode of inhibition as the best-fitting model, thereby implying that BTB-1 binds only to the complex formed by Mt-bound Kif18A. If this applies, then BTB-1 would not be expected to inhibit the basal, microtubule-independent ATPase activity of Kif18A. To test this, we used an enzyme-coupled assay to measure the basal ATPase activity of recombinant HisKif18A ${ }^{\text {motor }}$ before the addition of DMSO/BTB-1 (phase I), after the addition of DMSO/BTB-1 (phase II), or the stimulated ATPase activity of His-Kif18 ${ }^{\text {motor }}$ upon addition of Mts in the presence of DMSO or BTB-1 (phase III). To be able to measure the low, Mt-independent ATPase activity of Kif18A, we had to use high concentrations of His-Kif18A ${ }^{\text {motor }}$ and, accordingly, of BTB-1. Before the addition of BTB-1 (Figure $3 \mathrm{~d}$, blue line) or DMSO (Figure $3 \mathrm{~d}$, red line), HisKif18A ${ }^{\text {motor }}$ hydrolyzed ATP at a rate of approximately $0.12 \mathrm{~s}^{-1}$ (Figure $3 \mathrm{~d}$, phase I). ATP hydrolysis was mediated by His-Kif18A ${ }^{\text {motor }}$ as no ATP turnover was detectable in identically treated samples lacking recombinant motor protein (Figure 3d, violet (DMSO) and black (BTB-1) lines). Intriguingly, addition of $100 \mu \mathrm{M}$ BTB-1 did not affect the ATPase activity of His-Kif18A ${ }^{\text {motor }}$ in the absence of Mts (Figure $3 \mathrm{~d}$ and $3 \mathrm{f}$, phase II: $0.12 \mathrm{~s}^{-1}$ (BTB-1) and $0.11 \mathrm{~s}^{-1}$ (DMSO)). The transient increase in absorption observed upon BTB-1 addition was unrelated to Kif18A as the same effect was observed for the BTB-1 treated sample lacking Kif18 ${ }^{\text {motor }}$ (Figure $3 \mathrm{~d}$, black line, phase II). Importantly, BTB-1 significantly inhibited the Mt-stimulated ATPase activity of His-Kif18A $\mathrm{A}^{\text {motor }}$ (Figure $3 \mathrm{~d}$ and $3 \mathrm{f}$, phase III: $0.13 \mathrm{~s}^{-1}$ (BTB-1) and $0.3 \mathrm{~s}^{-1}$ (DMSO)) corroborating our model that the inhibitory activity of BTB-1 depends on the formation of complexes between Kif18A and Mts. Monastrol, consistent with previous reports, ${ }^{[6]}$ inhibited both the basal and Mt-stimulated ATPase activity of Eg5 (Figure 3e,g). Collectively, these data imply that BTB-1 inhibits Kif18A in an ATP-competitive, Mt-uncompetitive manner.

We tested whether BTB-1 affects the mitotic progression of HeLa cells. RNA-interference (RNAi)-mediated depletion of Kif18A causes severe defects in spindle morphology accompanied by decisive failures in chromosome congression resulting in the accumulation of cells at an early stage of mitosis. ${ }^{[2,7]}$ Notably, HeLa cells treated with BTB-1 accumulated in mitosis in a dose-dependent manner (Figure $4 \mathrm{c}$ ). Immunofluorescence images revealed that spindle structures were severely compromised in BTB-1 treated cells (Figure 4b). Yet, elongated spindles observed upon RNAi-

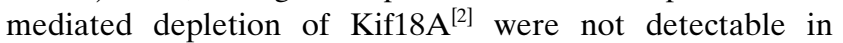
BTB-1 treated cells. However, the dual-functionality of Kif18A - it can move along Mts and depolymerize them at the tips-complicates the interpretation of phenotypes caused by unequal approaches, that is, removal of Kif18A from the cellular context by RNAi versus inhibition of its ATPase activity by BTB-1. Thus, further research efforts are required to unambiguously evaluate Kif18A as the relevant target of BTB-1 in cells.

In summary, we report herein the discovery of the first inhibitor of Kif18A, BTB-1, identified by a protein-based, 
a)
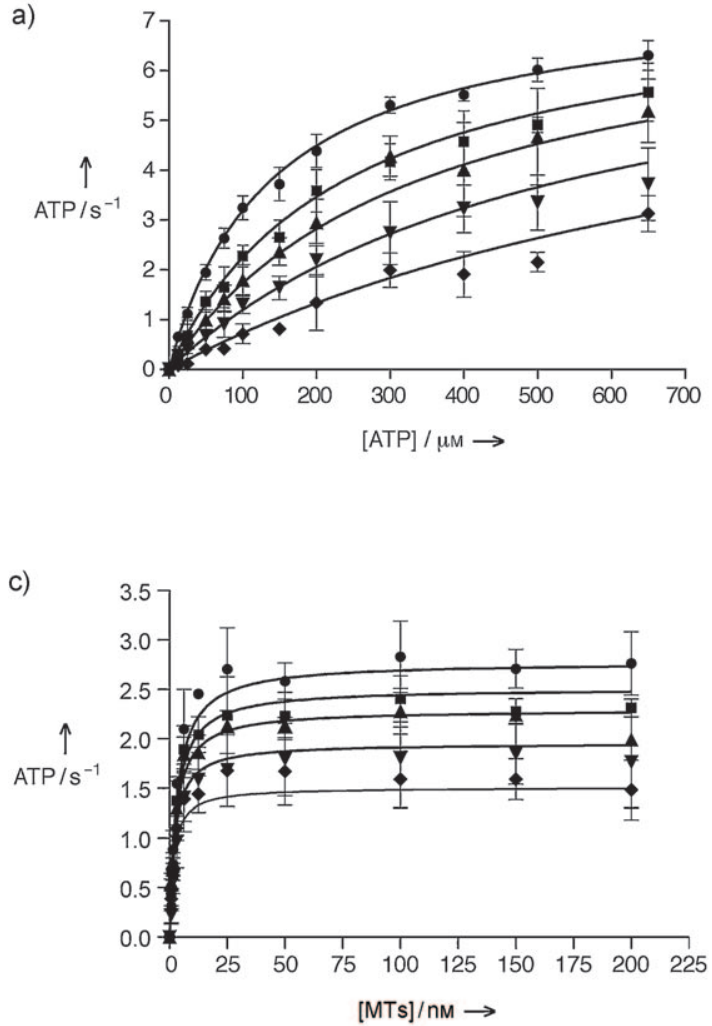

e)

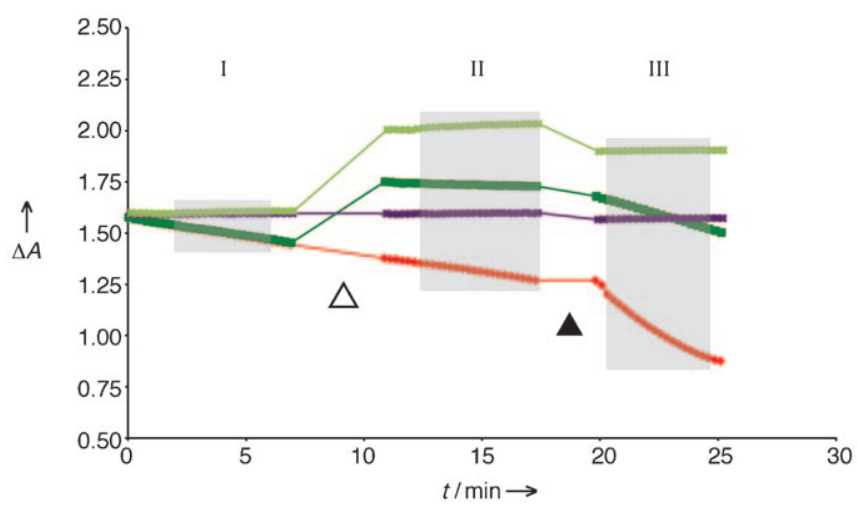

b)

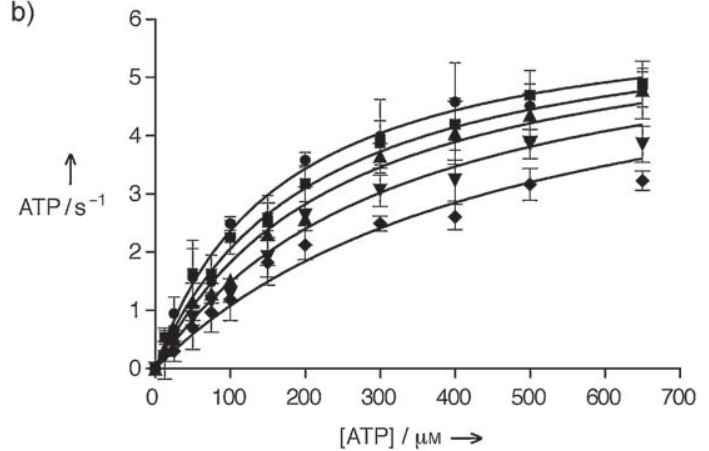

d)
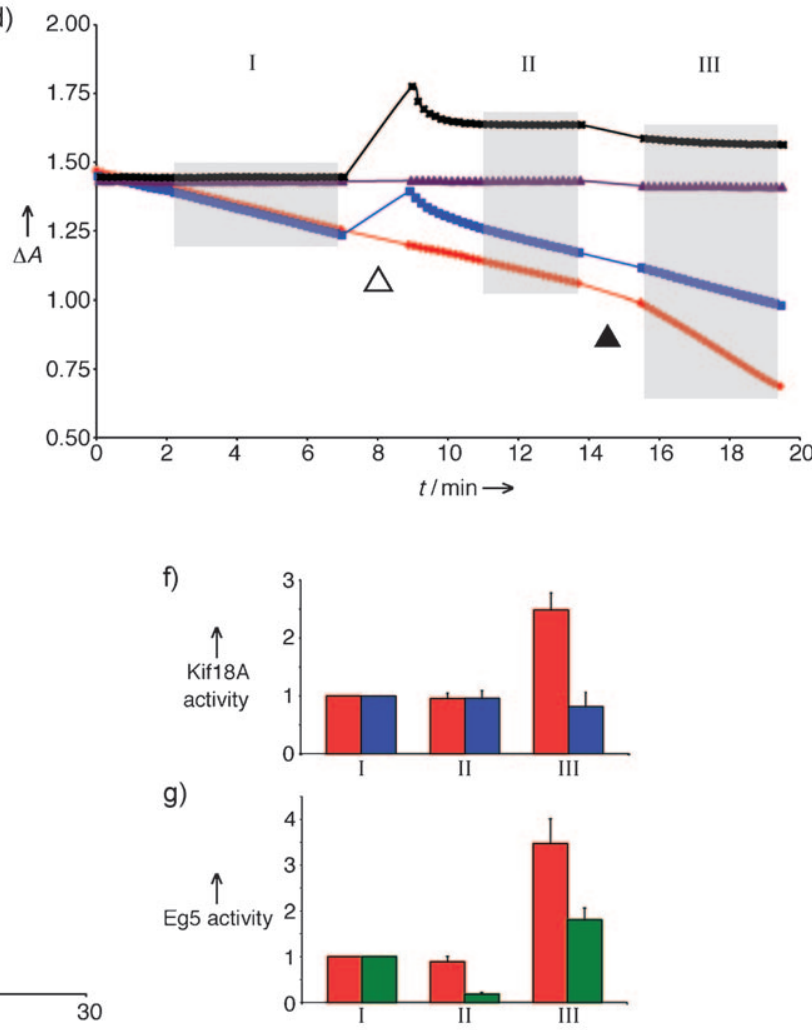

Figure 3. a) The activity of His-Kif1 $8 \mathrm{~A}^{\text {motor }}$ at increasing concentrations of ATP was monitored in the presence of $3 \mu \mathrm{M}$ Mts and increasing

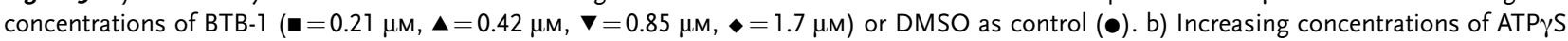
$(\boldsymbol{\|}=25 \mu \mathrm{M}, \boldsymbol{\Delta}=50 \mu \mathrm{M}, \boldsymbol{\nabla}=100 \mu \mathrm{M},=200 \mu \mathrm{M})$ or DMSO as control $(\bullet)$ were used as in (a). c) Increasing concentrations of BTB-1 $(\mathbf{-}=0.21 \mu \mathrm{M}, \mathbf{\Delta}=0.42 \mu \mathrm{M}, \boldsymbol{\nabla}=0.85 \mu \mathrm{M},-1.7 \mu \mathrm{M})$ or DMSO as control $(\bullet)$ were tested on His-Kif18A ${ }^{\text {motor }}$ at $650 \mu \mathrm{M}$ ATP and varying concentration of Mts. d) ATPase activity of $705 \mathrm{nM}$ His-Kif18A $\mathrm{A}^{\text {motor }}$. Phase I: basal ATPase activity before addition of DMSO (red line) or $100 \mu \mathrm{M}$ BTB-1 (blue line). Phase II: basal ATPase activity after addition of DMSO or $100 \mu \mathrm{M}$ BTB-1. Phase III: Mt-stimulated ATPase activity in the presence of DMSO or $100 \mu \mathrm{M}$ BTB-1. Violet line: DMSO control reaction lacking His-Kif18A ${ }^{\text {motor. }}$. Black line: BTB-1 control reaction lacking HisKif1 $8 \mathrm{~A}^{\text {motor }}$. $\triangle$ : DMSO/BTB-1 addition; $\mathbf{\Delta}$ : Mts addition; light-gray areas: time points used to calculate the ATP hydrolysis rate in each phase. e) ATPase activity of $800 \mathrm{~nm}$ His-Eg5 ${ }^{\text {motor. }}$. Red line: DMSO; Dark green line: $100 \mu \mathrm{m}$ monastrol; Violet line: DMSO control lacking His-Eg5 ${ }^{\text {motor }}$; Light-green line: monastrol control lacking His-Eg5 ${ }^{\text {motor }}$. f) Quantification of His-Kif18A ${ }^{\text {motor }}$ activity during phase I, II, and III as described in (d); averages of three independent experiments and standard deviations are shown; the quantification of the buffer controls is omitted; red=DMSO, blue = BTB-1. g) Quantification of His-Eg5 ${ }^{\text {motor }}$ activity, averages of three independent experiments and standard deviations are shown; the quantification of the buffer controls is omitted; red=DMSO, green $=$ monastrol.

reverse-chemical genetic screen. Detailed enzymatic studies revealed that BTB-1 is a potent inhibitor of Kif18A $\left(\mathrm{IC}_{50}=\right.$ $1.69 \mu \mathrm{M})$ that acts reversibly in an ATP-competitive and Mtuncompetitive manner, that is, BTB-1 competes with ATP for Kif18A binding only when the motor-protein is associated with its pseudosubstrate microtubules. This distinctive char- acteristic combined with its fast and reversible mode of inhibition makes BTB-1 a powerful tool to dissect the mechanochemical properties of Kif18A, a kinesin that integrates both microtubule motility and depolymerizing activity. Thus, our chemical-biology-based approach has provided access to a new tool for studying a protein key for 
a)

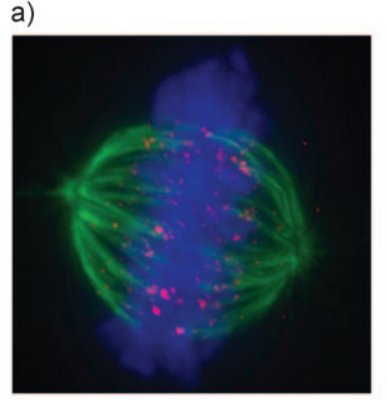

b)

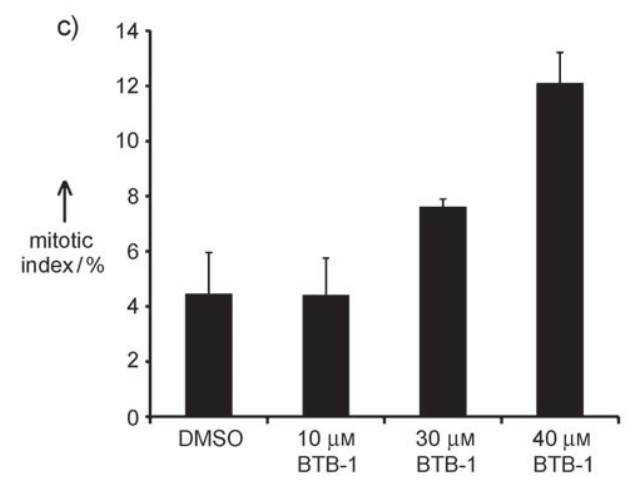

Figure 4. Immunostaining of HeLa cells treated for $18 \mathrm{~h}$ with a) DMSO or b) $30 \mu \mathrm{M}$ BTB-1. Red=Kif18A; green= microtubules; blue $=$ DNA (scale bar $=5 \mu \mathrm{m}$ ). c) Quantification of the mitotic index of HeLa cells treated with DMSO or increasing concentration of BTB-1 ( $n \geq 180$ cells; averages of three independent experiments and standard deviations are shown).

chromosome segregation in mammalian cells. Examples of inhibitors of the mitotic kinesin $\mathrm{Eg} 5,^{[5,8-10]}$ non-muscle myosin II ${ }^{[11]}$ and Polo-like $(\mathrm{Plk} 1)^{[12-15]}$ or Aurora ${ }^{[16]}$ kinases demonstrate that small molecules are not only invaluable probes for basic science, but can also open up new avenues in the treatment of mitosis-related diseases, such as cancer. Current efforts involve the analyses of the cellular effects of BTB-1.

Received: August 12, 2009

Published online: October 23, 2009
Keywords: antiproliferation - enzymes - inhibitors - mitosis · molecular motors

[1] T. Wittmann, A. Hyman, A. Desai, Nat. Cell Biol. 2001, 3, E28.

[2] M. I. Mayr, S. Hummer, J. Bormann, T. Gruner, S. Adio, G. Woehlke, T. U. Mayer, Curr. Biol. 2007, 17, 488.

[3] M. L. Gupta, Jr., P. Carvalho, D. M. Roof, D. Pellman, Nat. Cell Biol. 2006, 8, 913.

[4] V. Varga, J. Helenius, K. Tanaka, A. A. Hyman, T. U. Tanaka, J. Howard, Nat. Cell Biol. 2006, 8, 957.

[5] T. U. Mayer, T. M. Kapoor, S. J. Haggarty, R. W. King, S. L. Schreiber, T. J. Mitchison, Science 1999, $286,971$.

[6] Z. Maliga, T. M. Kapoor, T. J. Mitchison, Chem. Biol. 2002, 9, 989.

[7] J. Stumpff, G. von Dassow, M. Wagenbach, C. Asbury, L. Wordeman, Dev. Cell 2008, 14, 252.

[8] S. DeBonis, D. A. Skoufias, L. Lebeau, R. Lopez, G. Robin, R. L. Margolis, R. H. Wade, F. Kozielski, Mol. Cancer Ther. 2004, 3 , 1079.

[9] S. Hotha, J. C. Yarrow, J. G. Yang, S. Garrett, K. V. Renduchintala, T. U. Mayer, T. M. Kapoor, Angew. Chem. 2003, 115, 2481; Angew. Chem. Int. Ed. 2003, 42, 2379.

[10] N. Sunder-Plassmann, V. Sarli, M. Gartner, M. Utz, J. Seiler, S. Huemmer, T. U. Mayer, T. Surrey, A. Giannis, Bioorg. Med. Chem. 2005, 13, 6094.

[11] A. F. Straight, A. Cheung, J. Limouze, I. Chen, N. J. Westwood, J. R. Sellers, T. J. Mitchison, Science 2003, 299, 1743.

[12] P. Lénárt, M. Petronczki, M. Steegmaier, B. Di Fiore, J. J. Lipp, M. Hoffmann, W. J. Rettig, N. Kraut, J. M. Peters, Curr. Biol. 2007, 17, 304.

[13] U. Peters, J. Cherian, J. H. Kim, B. H. Kwok, T. M. Kapoor, Nat. Chem. Biol. 2006, 2, 618.

[14] W. Reindl, J. Yuan, A. Kramer, K. Strebhardt, T. Berg, Chem. Biol. 2008, 15, 459.

[15] A. Santamaria, R. Neef, U. Eberspacher, K. Eis, M. Husemann, D. Mumberg, S. Prechtl, V. Schulze, G. Siemeister, L. Wortmann, F. A. Barr, E. A. Nigg, Mol. Biol. Cell 2007, 18, 4024.

[16] C. Ditchfield, V. L. Johnson, A. Tighe, R. Ellston, C. Haworth, T. Johnson, A. Mortlock, N. Keen, S. S. Taylor, J. Cell Biol. 2003, 161, 267. 